\title{
EVOLUCIÓN DEL HUMOR EN LA MUJER: DESDE PRIMARIA A LA UNIVERSIDAD ${ }^{1}$
}

\author{
LAURA M. ${ }^{a}$ Aliaga AgUZA \\ Grupo GRIALE \\ Universidad de Alicante
}

Recibido: 15/09/2014

Aceptado: 24/11/2014

\section{Resumen}

El comportamiento de hombres y mujeres se desarrolla de forma diferente desde edades tempranas, adoptando roles diferentes que influyen en nuestra forma de apreciar el mundo. Del mismo modo que los hombres y mujeres poseen distintas visiones del mundo, también entienden de forma diferente el humor. Antiguamente se pensaba que las mujeres no entendían el humor (Lakoff, 1975), sin embargo, esta concepción está cambiando (Ervin-Tripp and Lampert, 1992, o Hay, 1995). El objetivo de este artículo es analizar la evolución del humor en la mujer desde la etapa escolar, concretamente $4{ }^{\circ}$ de primaria, hasta el primer año universitario. Para ello, nos basaremos en dos corpus creados por el grupo GRIALE de la Universidad de Alicante para comparar los mecanismos humorísticos utilizados por hombres y mujeres.

Palabras clave: Evolución, Humor, Mujer

\begin{abstract}
Human behavior in men and women develops in different way from very early ages, taking different roles that influence the way we see the world. As men and women have different sights of the world, they also understand humor in a different way. In the past, it was thought that women did not understand humor (Lakoff, 1975), nevertheless, this point of view is changing (Ervin-Tripp and Lampert, 1992, or Hay, 1995). The goal of this paper is to analyze humor evolution in women from their
\end{abstract}

1. Este artículo se integra en el Proyecto I+D FFI2012-30941 «Innovaciones lingüísticas del humor: géneros textuales, identidad y enseñanza del español».

Feminismo/s 24, diciembre 2014, pp. 221-242

DOI: $10.14198 / \mathrm{fem} .2014 .24 .10$ 
schoolchild, to their first university year. To do so, we will base on two corpus, created by GRIALE group from Alicante university, to compare humor mechanisms used by men and women.

Keywords: Evolution, Humor, Woman

Feminismo/s 24, diciembre 2014, pp. 221-242 


\section{Introducción}

El comportamiento entre hombres y mujeres se desarrolla de forma diferente desde edades muy tempranas, ya que, dependiendo de nuestro sexo, adoptamos roles diferentes que influyen en nuestra forma de apreciar el mundo. Este hecho condiciona nuestra vida desde antes del nacimiento, esto es, el género se empieza a desarrollar en el momento en que los padres conocen el sexo del bebé, puesto que no se compran las mismas cosas para un niño que para una niña (Jule, 2008: 6). Del mismo modo que hombres y mujeres poseen distintas visiones del mundo, también entienden de forma diferente el humor. Si seguimos a Lakoff (1975: 56), siempre se ha pensado que las mujeres no tienen sentido del humor y no entienden las bromas, puesto que no cuentan chistes y si lo hacen, suelen arruinar el punch line al mezclar el orden de los acontecimientos. Actualmente esta concepción está cambiando, ya que hay trabajos que demuestran que las mujeres poseen sentido del humor, pero es diferente al de los hombres (por ejemplo, Ervin-Tripp and Lampert, 1992; o Hay 1995).

Por su parte, Ojeda Alba (2010) señala que hay pocos estudios que se centren en la competencia humorística en niños y los que hay, se centran en la apreciación y no en la creación del humor, con alguna excepción como por ejemplo, Droz y Ellis, 1996. Por ello, realiza un estudio en el que pretende identificar tendencias y patrones de género de hombres y mujeres en la etapa escolar que estudian una segunda lengua. En este artículo se establecen varias conclusiones interesantes: a) El humor se utiliza más en la etapa secundaria que en primaria. Este hecho confirma la afirmación de Manke (1998: 363) que señala que los niños y adolescentes carecen de las habilidades cognitivas necesarias tanto para producir como para entender el humor; b) los chicos se sienten más cómodos al realizar bromas que las chicas, de ahí que las chicas lo utilicen menos y por este motivo se piense que las chicas carecen de humor; y, c) las mujeres se sienten restringidas por los roles de género debido a la educación que han recibido. 
Partiendo de este hecho, el grupo GRIALE² de la Universidad de Alicante se propuso estudiar las diferentes manifestaciones del humor en los niños de primaria en la segunda fase del proyecto I+D (FFI2012-30941) Innovaciones lingüisticas del humor: géneros textuales, identidad y enseñanza del español, dirigido por la doctora Ruiz Gurillo. Para ello se elaboraron unos cuestionarios donde los niños de cuarto de primaria (curso 2012-2013) de 5 colegios de la provincia de Alicante, debían escribir unos relatos humorísticos anónimos sobre un intercambio escolar en Marte. Este estudio se completará en el curso 2014-2015, creando otro modelo de relato humorístico para los alumnos de sexto de primaria, con el objeto de comprobar la evolución en la competencia humorística de los niños.

Por otra parte, tras el inicio de las investigaciones de género y humor con las narraciones de primaria, surgió otro proyecto, Red docente Humor y perspectiva de género: análisis y aplicaciones didácticas (2012-2013 Modalidad II), coordinado por Larissa Timofeeva. Con ello se pensó que era una buena manera de disponer de datos complementarios en alumnos universitarios. En este estudio se pedía a alumnos de la universidad de Alicante que escribiesen anécdotas humorísticas sobre malentendidos culturales de forma anónima.

En este artículo nos proponemos realizar un estudio contrastivo en el humor femenino desde la primera fase de la educación, concretamente cuarto de primaria, hasta el primer año de la etapa universitaria, con el objetivo de comparar el uso de una serie de marcas, indicadores e inferencias que se repiten en los cuestionarios creados en el seno del grupo GRIALE para los proyectos anteriormente citados. Por un lado, la elección de cuarto de primaria

2. El Grupo GRIALE según se presenta en su página web, http://dfelg.ua.es/griale/index. html, es el Grupo de Investigación sobre la ironía y el humor en español del Área de Lengua Española del Departamento de Filología Española, Lingüística General y Teoría de la Literatura de la Universidad de Alicante. Fundado en 2002, ha contado hasta el momento con tres proyectos $\mathrm{I}+\mathrm{D}$, así como con diversas ayudas de la Universidad de Alicante. En el proyecto actualmente en vigor (FFI2012-30941: «Innovaciones lingüísticas del humor: géneros textuales, identidad y enseñanza del español» participan también dos miembros externos de reconocido prestigio: Salvatore Attardo, de la Texas A \& M University-Commerce (Estados Unidos) y Javier Muñoz-Basols, de la University of Oxford (Gran Bretaña). Sus objetivos principales son el análisis pragmático de la ironía y el humor, la observación de aspectos socioculturales, como la incidencia del género en su empleo, y la aplicación de los resultados a la clase de español como lengua extranjera. Destacan como publicaciones conjuntas el libro de Leonor Ruiz Gurillo y Xose A. Padilla (eds.) (2009): Dime cómo ironizas y te diré quién eres. Una aproximación pragmática a la ironía. Frankfurt, Peter Lang; el trabajo colectivo del grupo GRIALE (2011): ¿Estás de broma? 20 actividades para practicar la ironía en clase de ELE. Madrid, Edinumen; y la obra de Leonor Ruiz-Gurillo y M. ${ }^{a}$ Belén Alvarado-Ortega (eds.) (2013): Irony and Humor: From Pragmatics to Discourse. Amsterdam, John Benjamins. 
viene suscitada debido a que a esta edad los niños ya son capaces tanto de crear como de comprender humor en distintos niveles, llegando al nivel del absurdo. Como señala Benavent (1970: 111-112):

Se alcanza la madurez de la comprensión cómica alrededor de los 10 años, al completarse con los contrastes entre causa y efecto y los contrastes con la realidad habitual; risas producidas por la proyección de situaciones de la vida cotidiana; risas producidas por la degradación de algo que somete u oprime (reglas, leyes y personas); gusto por lo cómico intelectual y el chiste; aumenta notablemente el número de gags cómicos que desencadenan la risa: estereotipos, inversiones, inferencias, etc.; risas provocadas por todo tipo de desviación de la normalidad; incipiente sentido del humor, tanto en el plano personal como en el interpersonal; control de las manifestaciones hiláricas a pesar de la comprensión del estímulo risible.

A partir de este momento el niño ha alcanzado la madurez cómica siendo capaz de controlar sus impulsos humorísticos y hacer reír a sus compañeros. Por otro lado, se ha elegido el primer año universitario, puesto que pensamos que es en esta etapa donde se alcanza la madurez tanto intelectual como social. Aquí ya se es capaz de tomar decisiones responsables y seleccionar el tipo de humor que hace reír al individuo. De este modo, podremos apreciar la evolución que ha tenido el humor desde el inicio de la madurez hasta su consolidación. Tras el análisis pretendemos contestar las siguientes cuestiones: ¿las mujeres son capaces de producir humor desde su infancia? ¿Existen mecanismos humorísticos que se utilicen en todas las etapas? ¿Se utilizan las inferencias, las marcas y los indicadores humorísticos de la misma forma en ambas etapas? ¿Está la mujer restringida por la educación -y no utiliza el humor-o es capaz de producir humor? La hipótesis de la que partimos es que las mujeres poseen humor a lo largo de toda su vida; sin embargo, no se ríen de las mismas cosas que los hombres, porque el ser humano está determinado humorísticamente, no por la educación, sino por el entorno, esto es, existe un cierto determinismo humorístico. Como muchos aspectos del desarrollo del ser humano, el humor viene determinado por las distintas experiencias tanto vitales como culturales. Por ello, hacemos humor de lo que conocemos, esto es, es el entorno el que nos determina sobre qué reír y hacer reír.

En este artículo en primer lugar explicaremos tanto el corpus de primaria (§ 2.1), como el universitario ( $\S 2.2$ ), utilizados para nuestro estudio. A continuación, nos centraremos en el análisis práctico (§ 3), donde compararemos dos indicadores lingüísticos (fraseología e hipérbole), una marca (exclamación) y dos inferencias humorísticas (choque cultural y situación ridícula) que se utilizan en ambos corpus y, así, llegaremos a las conclusiones pertinentes (§ 4). 


\section{Presentación del Corpus}

En este epígrafe nos centraremos en la explicación de los dos corpus de los que se han extraído los ejemplos seleccionados para este análisis cualitativo. Explicaremos cómo se han obtenido y cómo se han recopilado tanto los datos del corpus de primaria (§ 2.1), como los del corpus universitario (§ 2.2).

\subsection{Corpus de Primaria}

El Corpus de Primaria lo hemos extraído de un estudio relacionado con el Proyecto «Innovaciones lingüísticas del humor: géneros textuales, identidad y enseñanza del español» del grupo GRIALE, cuya investigadora principal es Leonor Ruiz Gurillo. El Grupo GRIALE de la Universidad de Alicante al que pertenecemos pretende investigar en la segunda fase del proyecto I+D mencionado si existen diferencias de uso del humor entre hombres y mujeres. Por ello, siguiendo la propuesta de Ojeda Alba (2010), se decidió comenzar por obtener narraciones de alumnos de $4 .^{\circ}$ de primaria, redacciones que se repetirán a los mismos alumnos en dos años académicos. En estas redacciones se pedía a los alumnos de $4 .^{\circ}$ de primaria de 5 colegios de la provincia de Alicante que escribiesen un relato humorístico de forma anónima sobre un intercambio escolar a Marte con el título Un cuento humorístico (Figura 1). Estos colegios son: Colegio Público «Divina Aurora» de Beneixama, Colegio Público «Mare de Déu del Carme» de Canyada, Colegio Público «Antonio Machado» de Elda, Colegio Público «Inmaculada Concepción» de Torrevieja y Centro Privado «San Alberto Magno» de Monforte del Cid.

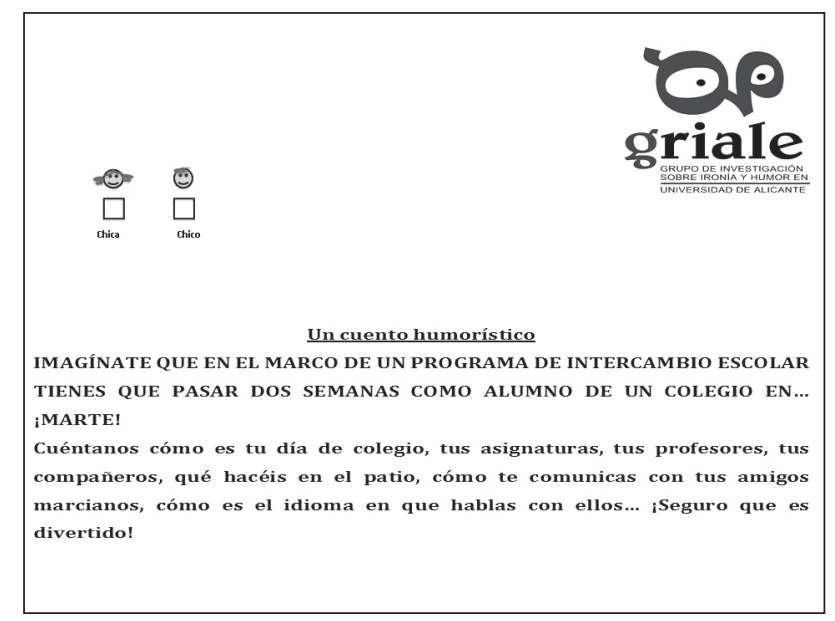

Figura 1: Modelo de redacción propuesta para los alumnos de $4 .^{\circ}$ de primaria. 
Tras la recogida de datos, se elaboró una base de datos con el programa Microsoft Access y posteriormente se exportaron los resultados al programa Microsoft Excel. Las variables que se tuvieron en cuenta para la elaboración de la base de datos fueron: sexo (femenino / masculino), tipo de centro (público / privado) y línea del centro (castellano / valenciano/ multilingüe). El procedimiento que se llevó a cabo para la recogida de datos se realizó en varias fases: en primer lugar, el grupo GRIALE se puso en contacto con los colegios seleccionados para el estudio y se estableció una fecha para la recogida de redacciones. En segundo lugar, se procedió al etiquetado de las redacciones. De esta manera, cada redacción tiene un código para poder identificarlas. La primera letra representa al colegio al que pertenece dicha redacción ( $\mathrm{A}=$ Colegio Público Antonio Machado, Elda; B = Colegio Público Divina Aurora, Beneixama; I = Colegio Público Inmaculada Concepción, Torrevieja; M = Colegio Público Madre de Déu, La Canyada; S = Centro Privado San Alberto Magno, Monforte del Cid), la segunda letra identifica el sexo del alumno (A niña y O niño) y, por último, aparece el número de redacción. De este modo, tendríamos que SA1, sería una redacción escrita por una niña del Centro Privado San Alberto Magno en Monforte del Cid. En tercer lugar, se realizó una lectura y volcado de la información, con la que se elaboró la base de datos.

Así, se obtuvieron 148 redacciones. Entre ellas 75 fueron escritas por niñas y 65 contienen humor, lo que representa un 43'92\%. Por otro lado, obtuvimos 72 narraciones escritas por niños, de las cuales 60 tienen humor, lo que representa un 40'54\%. En una de las redacciones no está indicado el sexo del alumno, porque se dejó la casilla correspondiente sin rellenar.

\section{Corpus Primaria}

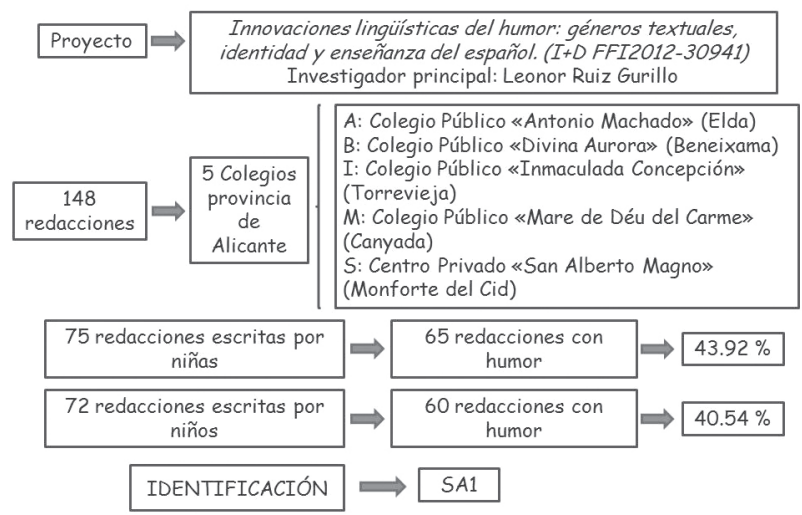

Figura 2: Cuadro-resumen de los participantes de $4 .^{\circ}$ de primaria

Feminismo/s 24, diciembre 2014, pp. 221-242 


\subsection{Corpus universitario}

El corpus universitario se ha extraído de un estudio relacionado con la Red docente humor y perspectiva de género: análisis y aplicaciones didácticas (20122013 Modalidad II) del grupo GRIALE, cuya coordinadora es Larissa Timofeeva. Tras el inicio de las investigaciones sobre género y humor con las narraciones de primaria, se creó un proyecto para recopilar datos complementarios sobre los recursos humorísticos utilizados por alumnos universitarios. Para este estudio se creó un cuestionario donde se pedía a alumnos universitarios que escribiesen de forma anónima anécdotas humorísticas que conociesen a

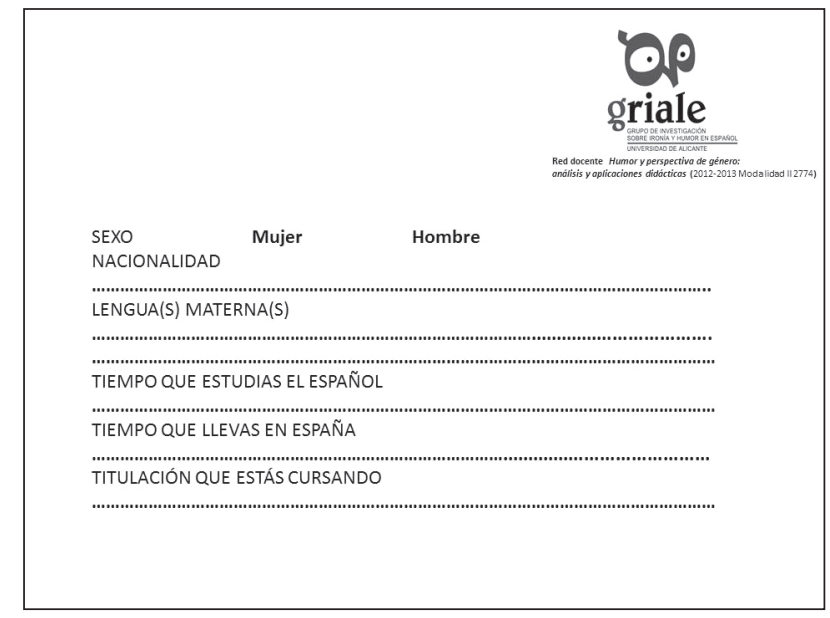

Un relato humorístico

En cada país hay costumbres y tradiciones que llaman la atención o pueden parecer extrañas para un extranjero. Muchas veces, son el origen de los tópicos y creencias, algunos verdaderos y otros no tanto. En el caso de España hay muchos ejemplos: en la película Misión Imposible 2 las Fallas valencianas se mezclan incomprensiblemente con la Semana Santa sevillana; muchos extranjeros creen que La Tomatina, que se celebra en Buñol, es una especie de fiesta nacional; o que la siesta es una costumbre a la que todos los españoles, sin excepción, dedican un par de horas al día; por no mencionar los casos "clásicos" del flamenco y los toros.

Sin embargo, cuando vamos al extranjero o nos comunicamos con las personas de otros países, nos damos cuenta de que con frecuencia los tópicos no son ciertos, lo cual puede provocar situaciones embarazosas o cómicas. Cuéntanos alguna historia que te haya pasado a ti o a alguien que conoces por culpa de una creencia falsa o un malentendido cultural.

Figura 3: Modelo de redacción propuesta para los alumnos universitarios.

Feminismo/s 24, diciembre 2014, pp. 221-242 
causa de una creencia falsa o malentendido cultural, bajo el título Un relato humorístico (Figura 3). Dichos alumnos son, por una parte, estudiantes de español de la universidad de Alicante con un nivel B2 y alumnos nativos pertenecientes tanto al grado de traducción como al de filología de dicha universidad.

A partir de los datos extraídos de los cuestionarios se elaboró una base de datos con el programa Microsoft Access y se exportaron los resultados al programa Microsoft Excel. Las variables que se consideraron para crear este corpus fueron: sexo (masculino / femenino), nacionalidad, lengua materna, tiempo que estudia español y tiempo que lleva en España. El procedimiento que se llevó a cabo para la elaboración de la base de datos fue el mismo que se realizó en la base de datos de primaria. En primer lugar, el grupo GRIALE se puso en contacto con los responsables de los departamentos de español, traducción y filología de la universidad de Alicante donde se pasó el cuestionario y se estableció una fecha límite de entrega. En segundo lugar, se creó el código de cada redacción para poder identificarlas. La primera letra corresponde a la nacionalidad ( $\mathrm{N}$ nacional y $\mathrm{E}$ extranjero), la segunda letra representa el sexo del participante (A chica y O chico) y, por último, se indica el número de redacción. Así tendríamos que NA53, sería una redacción escrita por un hablante nativo femenino.

De este modo, se obtuvieron 161 redacciones. Entre ellas 111 fueron escritas por hablantes nativos, concretamente 84 de ellas fueron redactadas por mujeres y 56 tienen humor, lo que representa un $366^{\prime} 65 \%$. Y 27 por hombres de las cuales 13 poseen humor, esto es, un $8^{\prime} 07 \%$.

\section{Corpus Universidad}

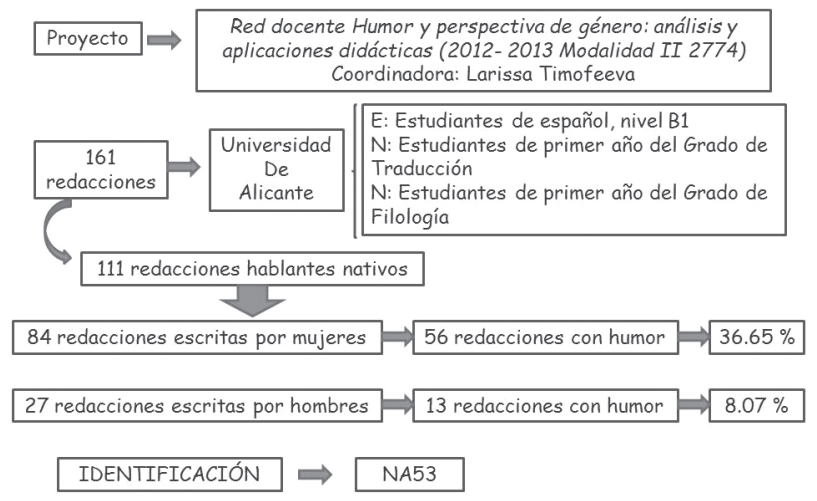

Figura 4: cuadro - resumen de los participantes universitarios.

Feminismo/s 24, diciembre 2014, pp. 221-242 


\section{Análisis}

En este apartado realizaremos el análisis práctico donde veremos, por una parte, dos indicadores (la fraseología y la hipérbole) y una marca (la exclamación) como elementos que identifican enunciados humorísticos y, por otra parte, dos inferencias humorísticas (el choque cultural y la situación ridícula) como formas de captar el humor. Todos estos mecanismos humorísticos se utilizan tanto en la fase primaria como en la fase universitaria. La selección de estas marcas, indicadores e inferencias se debe a que aparecen en una proporción similar en ambos corpus. El resto de elementos propuestos por GRIALE para la elaboración de la base de datos de ambos grupos de narraciones será analizado en trabajos posteriores.

Antes de comenzar con el análisis definiremos los mecanismos humorísticos seleccionados para facilitar la comprensión de los ejemplos. Ruiz Gurillo y Padilla García (2009) distinguen entre marcas e indicadores que ayudan a que el oyente / lector realice correctamente el proceso de inferencia en los enunciados irónicos. Partiendo de esta base amplían su objeto de estudio al humor (por ejemplo, Ruiz Gurillo, 2012; Ruiz Gurillo y Alvarado Ortega, 2013; Alvarado Ortega y Ruiz Gurillo, 2013). Siguiendo al grupo GRIALE entendemos por marca aquel elemento que aparece de forma escrita $u$ oral y ayuda a identificar un enunciado como humorístico, mientras que el indicador es un elemento humorístico en sí mismo. Ambos elemento ayudan al proceso cognitivo de inferencia para interpretar el humor, es decir, la inferencia es el proceso que lleva a cabo el oyente para concebir el enunciado como humorístico.

A continuación, analizaremos los mecanismos humorísticos seleccionados para este artículo. En primer lugar, prestaremos atención al indicador fraseológico ( $\$ 3.1$ ); en segundo lugar, observaremos el indicador hipérbole (§ 3.2); en tercer lugar, nos centraremos en la marca exclamación (§ 3.3); y, por último, las inferencias seleccionadas, esto es, las situaciones ridículas ( $\S$ 3.4.1) y el choque cultural (\$ 3.4.2)

\subsection{La fraseología como indicador humorístico}

En este epígrafe veremos unos ejemplos donde el indicador fraseológico es el encargado de crear el efecto hilarante. Se trata de un recurso bastante utilizado en ambos corpus, aunque más común en las redacciones universitarias, posiblemente debido al vocabulario más amplio que poseen dichos sujetos. Siguiendo al grupo GRIALE (Ruiz Gurillo y Padilla García, 2009), los indicadores son elementos que crean humor por sí mismos, esto es, poseen humor 
en su construcción interna, concretamente, el indicador fraseológico juega tanto con el significado literal de la expresión como con el idiomático para conseguir el efecto cómico.

A continuación, analizaremos cuatro ejemplos, dos casos extraídos del corpus de primaria y otros dos del corpus universitario, que representan la evolución que ha sufrido el uso de fraseología con intención humorística en la mujer.

\subsubsection{Corpus de primaria}

En este apartado comentaremos dos casos extraídos de las redacciones de primaria donde se utiliza alguna expresión fraseológica para crear el efecto cómico. En el primer caso (1), la niña está describiendo cómo eran sus profesores en Marte:

(1) «Mis profesores eran la bomba literalmente, cuando jugábamos a la bomba los profes se hacían una bola y jugábamos con ellos»

[SA9]

En este ejemplo, la niña ya posee conciencia del valor fraseológico, puesto que comprende que estas expresiones tienen tanto significado literal como figurado, siendo el más común el significado figurado. En este caso, el humor se crea a partir del significado literal de la expresión fraseológica «ser la bomba», además, para dejar claro que utiliza dicho significado utiliza el término «literalmente» que ayuda al efecto cómico y que indica que discrimina ambos significados. En el ejemplo (2), otra niña está describiendo cómo es el lenguaje en Marte y sus impresiones hacia este idioma:

(2) «Porque es que es como si me hablaras en chino no entiendo nada. Aunque si yo no entiendo nada mi profesor o me lo vuelve a explicar o colleja al canto»

[BA11]

En este caso, el humor comienza a partir de la comparación con el uso de la unidad fraseológica «hablar en chino» y termina con el fraseologismo «colleja al canto», donde la niña muestra de manera humorística que no entendía nada del idioma marciano y su profesor tenía que volver a explicárselo. En ambos casos se ha utilizado el significado idiomático de la expresión fraseológica y por ello el emisor no ha necesitado señalar que era de forma «literal» como ocurre en el ejemplo (1). Además, en gran número de los cuestionarios humorísticos de primaria encontramos esta conciencia lingüística que sirve 
como marco para crear humor, puesto que el hecho de no entender a los marcianos les resulta cómico a los niños.

\subsubsection{Corpus universitario}

En este epígrafe comentaremos dos ejemplos extraídos del corpus universitario en el que se utiliza el indicador fraseológico para la creación de humor. En estos ejemplos apreciamos la utilización de expresiones fraseológicas para realizar autohumor. Veamos el ejemplo (3) y (4) donde ambas chicas recurren a unidades fraseológicas para expresar sus sentimientos vergonzantes y, de este modo, crear el efecto cómico:

(3) «Hace varios años, cuando fui a Inglaterra me sentí como un mono de feria, como un motivo de diversión con temas como el de los toros o las sevillanas»

[NA140]

(4) «De repente, la mujer de la casa [...] me invitó a pasar a la cocina y vi en la cocina muchos alimentos encima de la mesa y una paella. [...] Después de mucha presión, porque todos los invitados no me quitaban ojos de encima y yo en muchos momentos no sabía ni qué hacer, la paella estaba hecha»

[NA148]

En el ejemplo (3) la protagonista de la historia está relatando distintas anécdotas de cómo fue su estancia en Inglaterra. Dicha estancia, en un primer momento resultó incómoda, debido a los estereotipos que se tienen de los españoles. Sin embargo, utiliza este hecho para crear humor a través del significado idiomático de la unidad fraseológica «ser un mono de feria» donde expresa sus sentimientos de forma cómica. En el ejemplo (4), de nuevo debido a los estereotipos que se tienen de los españoles, cuando la protagonista de la historia llegó a la casa en la que se iba a alojar, todos la estaban esperando para darle la bienvenida y la obligaron a hacer una paella. En esta situación incómoda, la protagonista se convierte en el blanco de la burla, puesto que no sabía cocinar. En el fragmento seleccionado lo que crea humor es el uso de la unidad fraseológica que incentiva el sentimiento de incomodidad de la joven.

\subsubsection{Conclusión}

En estos cuatro ejemplos apreciamos que tanto en la etapa de primaria como en la universitaria se utiliza el mismo recurso humorístico, esto es, las unidades fraseológicas, ya sea con su significado literal, ya sea con su significado idiomático. Sin embargo, el cambio se produce en el blanco de la burla. 
Mientras que en primaria se crea humor a partir de los demás, en la etapa universitaria se ríen de uno mismo realizando autohumor, convirtiéndose a sí mismos en el objetivo de las bromas.

\subsection{La hipérbole como indicador humorístico}

En este apartado analizaremos el indicador humorístico hipérbole. Como hemos señalado anteriormente el indicador humorístico es un mecanismo que posee humor en sí mismo. En este caso, a través de la hipérbole se exagera una situación o los rasgos de un personaje y así se consigue el efecto cómico.

\subsubsection{Corpus de primaria}

En general, este indicador es muy utilizado por los niños de primaria para describir tanto a sus profesores como a sus compañeros marcianos, como muestra el ejemplo (5), aunque también lo utilizan para indicar la gran dificultad del idioma como vemos en (6):

(5) «El profe podrido tiene 10 cabezas, 145 piernas, 500 bocas y 5525 orejas»

[IA20]

(6) «Huy, huy, huy, huy, esto no me lo enseñó ni en tres años»

[BA11]

En (5) se recurre a la exageración para describir a su profesor y es lo que crea el efecto cómico. Además, nos parece conveniente señalar, que en muchas ocasiones dichas descripciones vienen acompañadas de un dibujo. En (6) el humor también aparece a través de la exageración cuando el emisor intenta explicar lo difícil que es el idioma marciano. En este caso el blanco de la burla sería el profesor debido a que es incapaz de enseñar el idioma a la niña, en lugar de afirmar que no fue capaz de aprenderlo. A esto hay que añadirle la repetición onomatopéyica que introduce la hipérbole. Cabe resaltar que para los niños son los marcianos los que son diferentes, ya que no hemos encontrado ejemplos donde sean los marcianos los que se extrañen cuando observan el aspecto físico de los niños de la Tierra.

\subsubsection{Corpus universitario}

En este subapartado trataremos un ejemplo donde se utiliza la hipérbole como mecanismo humorístico. Se trata de un indicador poco utilizado en comparación con el uso que se realiza en primaria. La hipérbole se utiliza para mostrar 
el concepto que tienen los extranjeros de los españoles, siendo el español el blanco de la burla, como se muestra en (7):

(7) «Un joven estadounidense me dijo que seguro que a mí me gustaba el flamenco y que se me daba muy bien. Les contesté que ni sabía que ni me gustaba, no me creyeron y se pusieron a dar palmas. Les repetí que no me gustaba, que en realidad lo odiaba, alucinaron un poco, y a modo de broma, me dijeron que seguro que era la deshonra del país»

[NA75]

En (7) el efecto cómico aparece a través de la hipérbole que se utiliza en estilo indirecto donde los extranjeros piensan que la protagonista de la historia es la deshonra del país porque no le gusta el flamenco, todo un tópico fuera de nuestras fronteras. Así, se convierte en el blanco de la anécdota. Además, dicho indicador se introduce con la expresión «a modo de broma», la chica del ejemplo quiere dejar claro que el recurso que utiliza es para crear hilaridad. Por otra parte, nos parece conveniente señalar que el tópico de que los españoles tienen que saber flamenco se repite en muchas de las redacciones junto con otras marcas, indicadores e inferencias diferentes.

\subsubsection{Conclusión}

El indicador hipérbole es un recurso humorístico muy utilizado por las niñas de primaria para crear humor, sobre todo, para exagerar los rasgos físicos de los marcianos o la dificultad del idioma. Sin embargo, según se avanza en edad y madurez es un recurso que se usa en menor proporción.

\subsection{La exclamación como marca humorística}

En este epígrafe nos centraremos en la marca más utilizada en ambos corpus. Siguiendo al grupo GRIALE (Ruiz Gurillo y Padilla García, 2009), una marca es un elemento que ayuda a crear el efecto humorístico, pero no es humorístico es sí mismo. La exclamación ayuda al emisor a señalar una expresión que desea resaltar para que el receptor preste más atención a ese enunciado, puesto que hay alguna inferencia que debe realizar que sin esos signos no haría.

\subsubsection{Corpus de primaria}

Entre las marcas humorísticas que encontramos en ambos corpus, la más utilizada es la exclamación, siendo más numerosa en el corpus de primaria, que se utiliza para llamar la atención sobre algún aspecto. Además, nos parece 
conveniente señalar que siempre se utiliza junto a otro indicador o inferencia. Por ejemplo, en (8):

(8) «De repente solté i¿Qué?! Habláis mi idioma. Los marcianitos dijeron que pensaban que hablábamos raro, no como ellos. ¡Me quedé de piedra! ¡Pero si ellos eran los raritos!»

La niña utiliza la exclamación para mostrar su asombro a través de la unidad fraseológica «quedarse de piedra» cuando se percata de que para los extraterrestres es rara. El giro humorístico aparece cuando la niña matiza a través de la exclamación y el uso del diminutivo que realmente los raros son los extraterrestres y no ella. De este modo cambia el blanco de la burla, donde en un principio se centraba en ella, finalmente son los marcianos. En otro caso en el que encontramos la marca exclamación, (9), se utiliza para expresar que el idioma que usan los marcianos es muy complicado:

(9) «También lo que más me ha asombrado era el idioma en el que hablaban, ¡me ha costado un montón aprenderlo!, pero por lo menos soy bilingüe porque ¡sé español y marciano!»

[IA5]

En (9) en primer lugar se utiliza la exclamación para mostrar el esfuerzo que ha tenido que realizar la protagonista para poder aprender el idioma marciano. En segundo lugar, podemos comprobar la conciencia lingüística que tienen los niños de primaria, puesto que no le ha importado tener que aprender un idioma tan difícil, ya que esto la ha convertido en una persona bilingüe. Este hecho es el que crea el efecto cómico en el fragmento seleccionado.

\subsubsection{Corpus universitario}

Al igual que ocurre en el corpus de primaria, en el universitario la marca exclamación se utiliza para resaltar algún indicador o inferencia, es decir, con el uso de esta marca el emisor pretende llamar la atención sobre el lector para que sepa que se trata de un enunciado humorístico. En el caso (10) se trata de una situación vergonzante que le ocurre a la protagonista a causa de un malentendido cultural.

(10) «El día que una compañera mía que practicaba deporte conmigo me invitó a su casa. Como yo sabía que era rusa cuando entré en su casa me quité automáticamente los zapatos. Mi amiga no se dio cuenta de mi acción y cuando entré en el salón ¡vi que estaban sus padres y sus hermanos con zapatos!»

[NA144]

Feminismo/s 24, diciembre 2014, pp. 221-242 
La exclamación se utiliza para mostrar la situación embarazosa en la que se vio involucrada la protagonista, a través de la contradicción entre las costumbres que pensaba que tenían los rusos -quitarse los zapatos al entrar en casa-y lo que realmente sucedió -solamente ella iba descalza. De este modo, la protagonista se convierte en el blanco de la broma.

\subsubsection{Conclusión}

La exclamación es la marca humorística más utilizada tanto en el corpus de primaria como en el universitario, debido a que puede acompañar a cualquier indicador e inferencia que el emisor considere que debe resaltar para que el lector / oyente se percate del efecto cómico que se quiere transmitir. No obstante, aparece en mayor proporción en primaria debido posiblemente a que las universitarias dominan más signos gráficos que las niñas.

\subsection{Dos tipos de inferencias humorísticas}

En este apartado trataremos la inferencia, concretamente nos centraremos en dos tipos de inferencias que, en ocasiones, están muy relacionadas entre sí, esto es, las situaciones ridículas (§ 4.4.1) y el choque cultural (§ 4.4.2). Siguiendo a Grice (1975) entendemos por inferencia aquello que no se dice expresamente en el texto, pero se deduce del mismo. Desde nuestro punto de vista, el choque cultural podría considerarse un tipo dentro de las situaciones ridículas, ya que dichas situaciones comprometidas muchas veces aparecen debido a culturas diferentes en contacto.

\subsubsection{Las situaciones ridículas}

En este epígrafe mostraremos dos ejemplos, uno extraído del corpus de primaria (§ 3.4.1.1) y otro del corpus universitario (§ 3.4.1.2), donde el efecto humorístico se crea a partir de dos situaciones ridículas, en las que se ven involucradas las protagonistas de cada anécdota, que se producen por desconocimiento cultural sobre el país.

\subsubsection{Corpus de primaria}

En el corpus de primaria encontramos menos casos en los que se describan situaciones humorísticas a causa de una situación ridícula, puesto que las redacciones correspondientes a este corpus se centran, sobre todo, en la descripción de lo que las niñas ven a su alrededor. Sin embargo, podemos encontrar alguna situación ridícula como en (11): 
(11) «Resulta que no necesitábamos los trajes espaciales y un alumno marciano me abrió la cápsula y yo como una tonta haciendo como si me ahogaba»

[SA9]

En este ejemplo, la niña desconoce que no necesita un traje especial para poder respirar en Marte; de ahí que, cuando se lo quitan, tenga la sensación de que se ahoga, hasta que se da cuenta de que sí puede respirar, y está haciendo el ridículo. Hemos elegido este ejemplo, para mostrar que, en ocasiones, en primaria, los niños también pueden ser el blanco de la burla, pero, además de no ser lo más habitual, ha llegado a esa situación por culpa del marciano que es el que le quita el traje.

\subsubsection{Corpus universitario}

En el corpus universitario encontramos más proporción de situaciones ridículas que en el de primaria, puesto que el tema de las redacciones de este grupo invita a describir situaciones embarazosas que posteriormente terminan convirtiéndose en anécdotas cómicas sobre una experiencia personal. El ejemplo (12) muestra una anécdota de una chica la primera vez que visitó Londres:

(12) «Cuando el taxi llegó fui a subirme por la puerta derecha del coche y como estaba tan nerviosa llegué a sentarme en el asiento y al segundo me di cuenta de que estaba sentada en el lado del volante»

[NA138]

La chica vuelve a ser el blanco de la burla, puesto que no se da cuenta de que en Inglaterra el volante está en el lado contrario. En este caso, la protagonista explica que realmente no fue desconocimiento de la cultura inglesa, sino que la confusión se creó por los nervios que tenía por ser la primera vez que visitaba la ciudad. De ahí que se vea involucrada en una situación vergonzante para ella, pero cómica para los demás.

\subsubsection{Conclusión}

Las situaciones ridículas son un recurso muy utilizado a la hora de crear humor, debido a que los errores de los demás suelen causar gracia en la sociedad española. Los ejemplos que hemos mostrado de situaciones ridículas dentro de este epígrafe, desde nuestro punto de vista, están muy relacionados con el choque cultural que veremos en el siguiente apartado, puesto que el efecto humorístico se produce debido a situaciones derivadas de diferencias culturales y no a una situación ridícula universal como puede ser un tropiezo o una caída. 


\subsubsection{El choque cultural}

Por último, en este subapartado nos centraremos en dos casos humorísticos a partir de la inferencia producida por el choque entre culturas que poseen costumbres diferentes, muy relacionada con los casos anteriores. El primero (§ 3.4.2.1) lo hemos extraído del corpus de primaria, mientras que el segundo (§ 3.4.2.2) del universitario.

\subsubsection{Corpus de primaria}

En este corpus encontramos muchos casos donde el efecto cómico se consigue a través del choque cultural, ya que las redacciones se centran en los diferentes aspectos que los niños han encontrado en el nuevo planeta que están visitando. El primer ejemplo que presentamos corresponde al corpus de primaria, (13), donde la niña explica cómo era su colegio en Marte:

(13) «Entré en mi clase y la clase era rarísima los muebles boca abajo, las mesas tenían pinchos»

[SA5]

Encontramos dicho choque cultural en la descripción que se realiza sobre el aula del colegio marciano. La discrepancia humorística se anuncia a través del superlativo «rarísimo». Se trata de un coche cultural humorístico, porque en nuestro país los muebles no se colocan de ese modo y, además, las mesas son cómodas para poder estudiar, al contrario que ocurre en el ejemplo seleccionado.

\subsubsection{Corpus universitario}

$\mathrm{Al}$ igual que ocurre con las situaciones ridículas, el choque cultural es una inferencia muy utilizada en este corpus debido al tema propuesto en la redacción. En el ejemplo (14), la chica está relatando una anécdota que le ocurrió a su abuelo hace varios años cuando vinieron a España unos amigos de Perú:

(14) «Mi abuelo hace unos años recibió la visita de unos peruanos. Un día iba paseando con ellos y pasaron por un kiosco en el que se hacen apuestas de fútbol. La hija de los peruanos gritó:

-Papá, mira ¡LA PICHA, LA PICHA!

El padre le contestó a la hija:

-No digas eso, aquí «picha» significa otra cosa»

[NA78]

En (14) encontramos el choque cultural a partir de las connotaciones diferentes que hay en el significado de una palabra entre el español de la península y 
el español de América. Concretamente, el efecto humorístico aparece a través de la palabra «picha» que en América es un término coloquial para designar a un juego de azar, mientras que en la península se trata de un eufemismo para nombrar al miembro reproductor masculino. Como ocurría en (11), hemos elegido este ejemplo, para mostrar que las universitarias también sitúan el blanco de la burla en otras personas, aunque no es lo más habitual.

\subsubsection{Conclusión}

Este tipo de inferencia es un recurso al que se recurre mucho en ambos corpus para crear el efecto hilarante, siendo más utilizado entre las universitarias que entre las niñas de primaria, posiblemente debido a que las universitarias poseen más conciencia lingüística y cultural que las niñas.

\section{Conclusiones}

Después del análisis efectuado podemos extraer una serie de conclusiones. El corpus muestra que las mujeres son capaces de crear humor desde las primeras fases de la educación. Por tanto, podemos afirmar, por una parte, que las niñas poseen las capacidades cognitivas necesarias para ello y que posteriormente las irán perfeccionando y, por otra parte, que las mujeres no se encuentran restringidas humorísticamente por la educación. No obstante, el humor en cada etapa del desarrollo humano es diferente. Existe un cierto determinismo humorístico, por un lado, el emisor crea humor del entorno, esto es, de las cosas que se conocen; y, por otro lado, se crean situaciones humorísticas por desconocimiento, ya sea cultural o verbal. También aparecen dichas situaciones humorísticas gracias a que el emisor aprecia que se trata de una situación cómica.

Como hemos visto en el análisis práctico, existen una serie de mecanismos humorísticos que se utilizan en todas las fases de la vida como, por ejemplo, los que hemos presentado en este artículo, a saber: indicadores como la fraseología y la hipérbole, la marca exclamación y las inferencias que hemos denominado situación ridícula y choque cultural.

En cuanto a la evolución del uso de estos mecanismos podemos apreciar una tendencia hacia el autohumor. En las primeras etapas de la vida se suele crear humor a partir de los demás, siendo en muy pocas ocasiones una misma el blanco de la burla. En la etapa universitaria el objetivo de la broma es el propio emisor, esto es, autohumor. Si nos centramos en cada mecanismo presentado de forma individualizada, podemos apreciar que en primaria, además de conocer algunas expresiones idiomáticas, ya distinguen entre el significado 
literal y el idiomático. Además, en esta etapa poseen conciencia lingüística, porque el hecho de no entender a los marcianos crea humor. En la etapa universitaria este indicador tiende a utilizarse para crear autohumor, para describir situaciones vergonzantes, debido a lo que los extranjeros piensan de los españoles. El indicador fraseológico se utiliza más en la etapa universitaria que en primaria. Sin embargo, el indicador hipérbole es más utilizado en primaria. En este caso se usa tanto para describir a los marcianos como para señalar la dificultad que presenta el idioma. Podemos apreciar que los enunciados humorísticos tienden a describir cómo los niños ven a los marcianos, mientras que en la etapa universitaria las anécdotas versan, en gran medida, sobre el concepto que los extranjeros tienen de los españoles.

En lo referente a la marca más utilizada en ambos corpus, la exclamación, existe un mayor uso por parte de las niñas que de las universitarias, debido, sobre todo, a que estas últimas dominan más signos gráficos. Este recurso siempre se utiliza junto con otros mecanismos, puesto que las marcas ayudan a identificar el humor, pero no son humorísticas en sí mismas. En ambos corpus destaca el uso junto al choque cultural: como la rareza de los extraterrestres y la dificultad de su idioma, en primaria; y situaciones vergonzantes para la protagonista de la historia, en la etapa universitaria. En lo que concierne a las inferencias hay una tendencia diferente de uso entre ambos corpus. Mientras que en primaria se recurre al choque cultural para crear el efecto cómico, en la etapa universitaria abusan de las situaciones ridículas. Esto puede ser debido a dos motivos fundamentales, en primer lugar, porque las situaciones ridículas es un tipo de humor que causa gracia en la sociedad española y en la infancia aún no se tiene conciencia del ridículo; y, en segundo lugar, por el tema propuesto en las redacciones, ya que en el cuento de primaria encontramos la descripción de muchos lugares distintos a la Tierra, cosa que no ocurre en las redacciones universitarias que se centran en las anécdotas vividas. Además, en las inferencias apreciamos un uso distinto al resto de los mecanismos utilizados, a pesar de no ser el más habitual: en las situaciones ridículas hemos encontrado algún caso, como el ejemplo que se ha utilizado en el análisis, donde la niña se convierte en el blanco de la burla; en el choque cultural aparece algún fragmento en el que el blanco de la burla se sitúa en otra persona. La figura 5 muestra el uso de los mecanismos presentados en este artículo. 


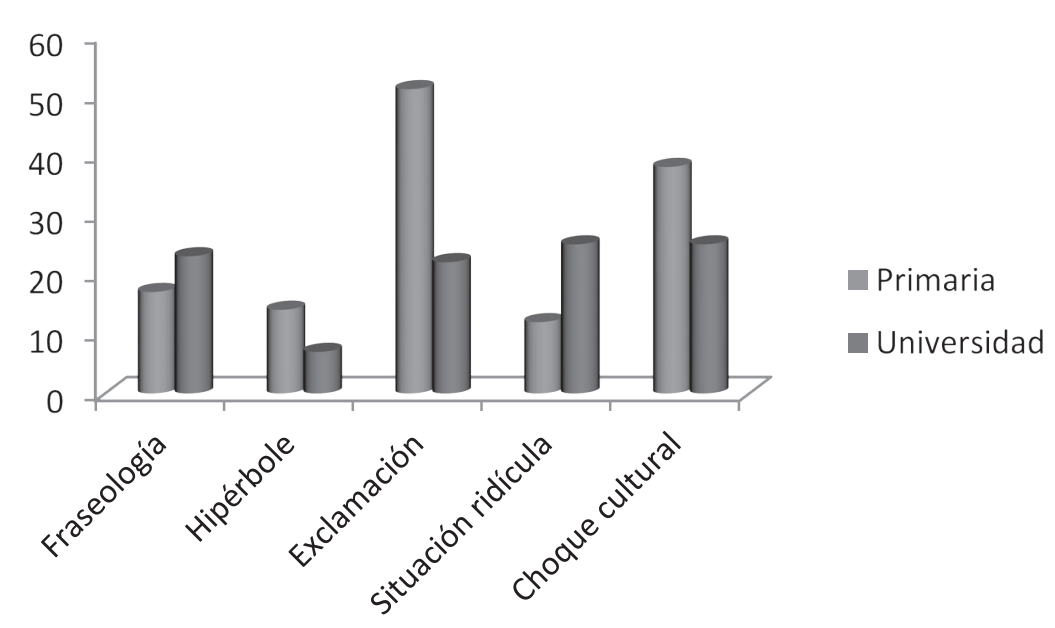

Figura 5: Mecanismos humorísticos

El trabajo aquí presentado es una primera aproximación al corpus de narraciones humorísticas de mujeres de primaria y de universidad. Se ha presentado un análisis cualitativo que cabrá ampliar en futuras investigaciones y corroborar a partir de datos cuantitativos, extendiendo el análisis a los datos obtenidos del sexo masculino.

\section{Referencias bibliográficas}

Alvarado Ortega, M. Belén y Ruiz Gurillo, Leonor (coords.). Humor, Ironía y Géneros Textuales. Alicante, Servicio de Publicaciones de la Universidad de Alicante, 2013.

Benavent Oltra, José Antonio. «Génesis de la risa infantil». Anuario de Psicología, Número 2 (1970), pp. 107-114.

Droz, Marilyn y ELLIS, Lori. Laughing While Learning: Using Humor in the Classroom. Longmont, Sopris West, 1996.

ERVIN-TRIPP, Susan and LAMPERT, Martin. «Gender differences in the construction of humorous talk» en Hall, Bucholtz and Moonwomon (eds.) Proceedings of the second Berkeley Women and Language, Berkeley, Women and Language Group, 1992, pp. 105-107.

GRICE, H. P. «Logic and Conversation» en Cole, P. and Morgan, J. L. (eds.) Speech Acts, New York, Academic Press, 1975, pp. 41-58.

HAY, Jennifer. Gender and humour: Beyond a joke. Master's thesis. Wellington, Victoria University of Wellington, 1995. 
JulE, Allyson. A Beginner's Guide to Language and Gender. Clevedon, MM Textbooks, 2008.

LAKOFF, R. Language and Woman's Place. New York, Harper and Row, 1975.

MANKE, B. «Genetic and environmental contributions to children interpersonal humor» en Ruch (ed.) The Sense of Humor Explorations of a Personality Characteristic, New York, Mounton de Gruyter, 1998, pp. 361-385.

OJEDA AlBA, Julieta. «Humour, Vocabulary and Individuality in an EFL Task», en Gendered Words: Representation and Identities, 2010, pp. 212-237.

RuIz GURILlo, Leonor. La lingüística del humor en español. Madrid, Arco Libros, 2012

Ruiz Gurillo, Leonor y Alvarado Ortega, M. Belén (eds.) Irony and Humor. From pragmatics to discourse. Ámsterdam, John Benjamins, 2013.

Ruiz Gurillo, Leonor y Padilla García, Xose. (eds.). Dime cómo ironizas y te diré quién eres. Berlín, Peter Lang, 2009. 